\title{
Evaluation of Lateral Deviation of Distribution Dose of Therapeutic Proton Beams in Voxelized Water Phantom Using the Monte Carlo Simulation Platform GEANT4
}

\author{
Othmane KAANOUCH ${ }^{1,2}$, Mustapha KRIM ${ }^{1}$, El Madani SAAD ${ }^{1}$, Ismail GHAZI ${ }^{3}$, Kamal SAIDI ${ }^{1,2}$, \\ Zineb SOBHY ${ }^{3}$, Meriem TANTAOUI ${ }^{3}$ \\ ${ }^{1}$ Hassan First University of Settat, High Institute of Health Sciences, Laboratory of Sciences and Health Technologies, BP \\ 555, 26000, Settat, Morocco \\ ${ }^{2}$ Department of Radiotherapy, Sheikh Khalifa International University Hospital, BP 82403, Casablanca, Morocco \\ ${ }^{3}$ Subatomic Research and Applications Team, Department of Physics, LPMC-ERSA, Faculty of Sciences Ben M'Sik, Hassan \\ II University, Casablanca, Morocco \\ othmane.kaanouch@gmail.com, mustapha.krim@uhp.ac.ma
}

\begin{abstract}
Proton therapy as Radiotherapy a treatment modality to treat cancer due to the favorable ballistic properties of proton beams. The proton loses the majority of its energy in biological matter by interaction with the electrons of molecules by an inelastic - coulomb - process which is bigger and maximal in the end of the range causing ionization or excitation, also through the interaction with the nucleus which is a minimal process in biological matter. Otherwise the last process is the responsible of the lateral deviation of the protons from the center. Our work consists to calculate and evaluate the lateral scattering of the dose distribution of protons acceleration up to energies within the therapeutic window between 70 and $250 \mathrm{MeV}$ using a voxelized water phantom as a 3D detector which detect and calculate the beam deposed energy and dose in any area of water phantom using Geant4 platform based on Monte-Carlo Method simulation. Results of our work are compared to the experimental references results and also to other methods of simulation results.
\end{abstract}

Keywords : Proton Therapy, Lateral Deviation, Voxelization, Distribution Dose, Monte Carlo 